\title{
Brownian Movement of Inorganic Nanoparticles in Sediments
}

\author{
P. FORNAL ${ }^{a}$ AND J. STANEK ${ }^{b}$ \\ ${ }^{a}$ Institute of Physics, Cracow Technical University, Podchorążych Str. 1, 30-083 Kraków, Poland \\ ${ }^{b}$ Marian Smoluchowski Institute of Physics, Jagiellonian University, Reymonta Str. 4, 30-059 Kraków, Poland \\ The $100 \mathrm{~nm}$ particles of $\mathrm{Fe}_{2} \mathrm{O}_{3}$ and metallic Fe sedimented jointly with $\mathrm{Al}_{2} \mathrm{O}_{3}$ powder from their suspension \\ in oleic acid exhibit distinguished mobility which depends on the concentration of aluminum oxide. This \\ observation is interpreted as the result of the interparticle $\mathrm{Fe}-\mathrm{Fe}$ magnetic interactions which lead to the formation \\ of the rigid network of magnetic metallic iron nanoparticles.
}

PACS: 05.40.Jc, 64.75.Xc, 47.57.ef, 66.30.Pa, 87.64.kx

\section{Introduction}

In the seventies of the previous century physicists partly lost their interests in Brownian movement. Now this topic come back to the frontier of research because of its connection to the nano-bio-technology and generally to the life science as the possible explanation of the speeding up of the transport phenomena at the cellular level [1]. The proper description of the nanoparticles mobility is crucial in molecular engineering [2], as well as in pharmacology [3] and catalysis [4].

The most popular and probably most effective experimental technique for the study of mobility of nanoobjects in soft matter is dynamic light scattering [5]. Its main advantage is the wide range of time windows which may be applied and the broad variety of the particles which may be studied. The limitation is that the environment must be transparent and, which is probable the crucial problem, the method supplies the average mobility of different particles in the distinguished localization in the system. The peculiarities of the Mössbauer spectroscopy, which is used in this work, are just opposite. The method is restricted, in practice, to iron bearing particles which displacements are measured in the characteristic time window of $141 \mathrm{~ns}$, the life time of the excited nuclear level of ${ }^{57} \mathrm{Fe}$. However, because of the sensitivity to the hyperfine interactions, the method distinguishes different particles. Moreover, it is possible to observe different localizations of the nanoobjects [6], characterized by the local viscosity. The studied specimens may be quite dense [7] and the optical transparency is not required [8].

The individual iron bearing nanoparticle may be treated as a classical thin absorber for which the Mössbauer absorption line has the Lorentzian shape $L\left(v_{r}\right)$, where $v_{r}$ is the relative particle - source velocity $v_{r}=$ $v-V$, where $v$ and $V$ is the source and particle velocity in the laboratory coordination system, respectively. The shape of the experimental absorption line $I(v)$ is the integral over all particles.

$$
I(v)=\int p(V) L(v-V) d V
$$

where $p(V)$ is the probability density that a nanoparticle experiences the velocity $V$, or just the velocity distribution profile. In the case where $p(V)$ is the sum of Gaussian, characteristic for different particle surroundings, the experimental line is the sum of Voigt lines.

The work is aimed at the demonstration of the possibility of this technique for the determination of velocity distribution profiles of nanoobjects. The studied system, the soft sediments of metallic iron, hematite and aluminum oxide in oleic acid probably has no practical application. However, from the methodological and the fundamental research point of view it appears interesting to determine the Brownian mobility of iron bearing nanoparticles, here $\mathrm{Fe}$ and $\mathrm{Fe}_{2} \mathrm{O}_{3}$, strongly interacting with each other and with separating them oil and $\mathrm{Al}_{2} \mathrm{O}_{3}$ particles.

\section{Samples}

Nanoparticles of Fe and $\mathrm{Fe}_{2} \mathrm{O}_{3}, 100 \mathrm{~nm}$ in diameter were suspended in colloid of $\mathrm{Al}_{2} \mathrm{O}_{3}$ particles in oleic acid as surfactant. The volume ratio of $\mathrm{Fe}: \mathrm{Fe}_{2} \mathrm{O}_{3}: \mathrm{Al}_{2} \mathrm{O}_{3}$ was $1: 2: x$, where $0 \leq x \leq 75$. The amount of $\mathrm{Fe}$ and $\mathrm{Fe}_{2} \mathrm{O}_{3}$ in each sample was $7.3 \mathrm{mg} / \mathrm{cm}^{2}$, and $10.5 \mathrm{mg} / \mathrm{cm}^{2}$, respectively, optimal for Mössbauer spectroscopy. The liquid was placed in plexi, thin wall container which served as sample holder. The amount of oleic acid has been chosen in such a way, that after sedimentation it formed a 1 $\mathrm{mm}$ thick particle free surface layer.

The 1024 channel transmission Mössbauer spectra were recorded in vertical geometry, at room temperature in the broad, from -15 to $15 \mathrm{~mm} / \mathrm{s}$, velocity range.

\section{Results}

The obtained Mössbauer spectra are displayed in Fig. 1. They consist of two Zeeman patterns correspond- 


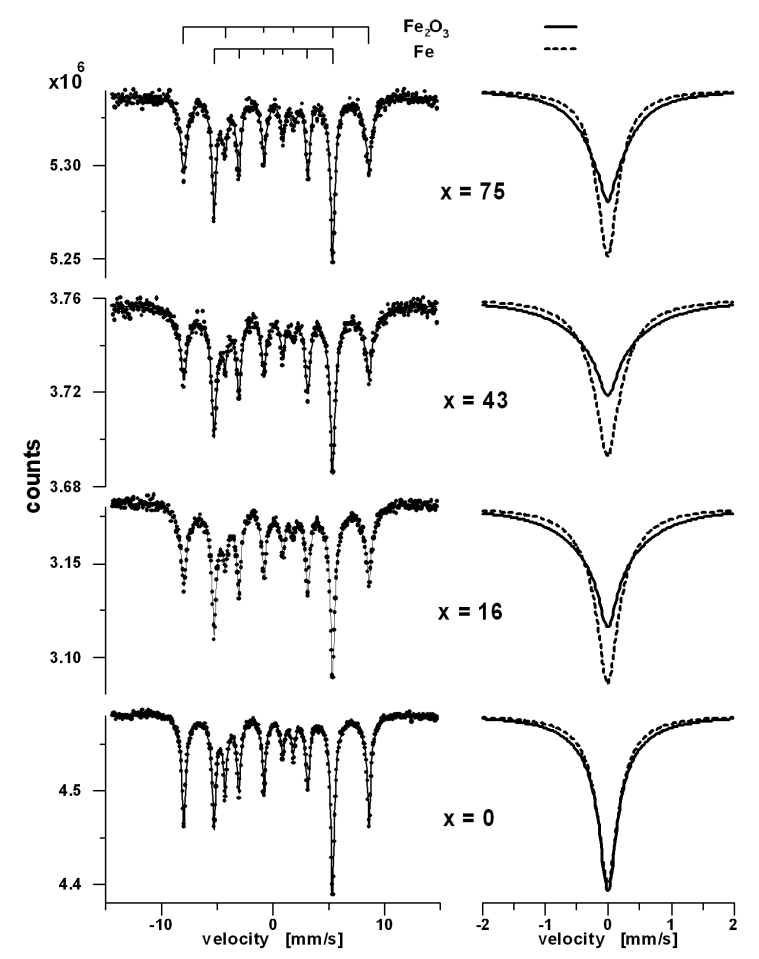

Fig. 1. Selected Mössbauer spectra of $\mathrm{Fe}, \mathrm{Fe}_{2} \mathrm{O}_{3}$, $\mathrm{Al}_{2} \mathrm{O}_{3}$ sediments in oleic acid with volume ratio 1:2:x for different $\mathrm{x}$. The $\mathrm{Fe}$ and $\mathrm{Fe}_{2} \mathrm{O}_{3}$ line position are marked. Right, the corresponding absorption line profiles.

ing to $\mathrm{Fe}$ and $\mathrm{Fe}_{2} \mathrm{O}_{3}$. Although the line positions are well known from the spectra of the initial materials pressed into pellets, the numerical analysis of the data was complicated. Because the information on he mobility of the particles may be concluded from the shape and broadening of the experimental resonance absorption line, the problem of the nonlinear background is crucial. Thus, at first the spectra were fitted with 12 Voigt lines with fixed position plus parabolic background. Next, using the parameters of the backgrounds, the spectra were corrected to the linear background and re-fitted using Zeeman patterns with fixed the known hyperfine parameters. It turned out that satisfactory fits with $\chi^{2}$ of 1.1 to 1.3 could be obtained only if we used four Zeemans with different line widths: two for $\mathrm{Fe}$ and two for $\mathrm{Fe}_{2} \mathrm{O}_{3}$. The corresponding line shapes for $\mathrm{Fe}$ and $\mathrm{Fe}_{2} \mathrm{O}_{3}$ nanoparticles in sediment are shown in Fig. 1, right. The lines show significant broadening, exceeding up to 5 times the natural line width. The broadenings, calculated as weighted average for each line, are shown in Fig. 2 in function of the volume concentration $\mathrm{x}$ of $\mathrm{Al}_{2} \mathrm{O}_{3}$.

\section{Discussion}

The main experimental facts are as follows:

1) The nanoparticles in the studied sediment exhibit Brownian movement; the velocity $v$ of their displacements within time $\tau, \tau=141 \mathrm{~ns}$, are up to

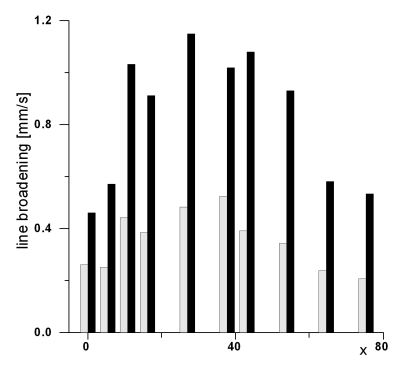

Fig. 2. The broadening of the absorption lines of metallic $\mathrm{Fe}$ (grey bars) and $\mathrm{Fe}_{2} \mathrm{O}_{3}$ (black bars), calculated as weighted average for each line, as a function of the volume concentration $\mathrm{x}$ of $\mathrm{Al}_{2} \mathrm{O}_{3}$.

$1 \mathrm{~mm} / \mathrm{s}$, it means that the displacements $\sigma, \sigma=v \tau$ of about $10^{-3} \mathrm{~m} / \mathrm{s} \times 1.41 \times 10^{-7} \mathrm{~s} \approx 10^{-10} \mathrm{~m}=$ $0.1 \mathrm{~nm}$ are observed.

2) The mobility of the $\mathrm{Fe}_{2} \mathrm{O}_{3}$ particles is twice as big as the mobility of the Fe particles.

The trivial reason of this might be some aggregation of magnetic nanopaticles. However, this does not explain the next fact, No. 3 .

3) The mobility of iron bearing nanoparticles is influenced by adding $\mathrm{Al}_{2} \mathrm{O}_{3}$ particles. It increases up to $x=30$ (90\% vol.) and next decreases. Probably this is the most intriguing fact. It means that $\mathrm{Fe}-\mathrm{Fe}$ interaction leads to stronger micro friction than in $\mathrm{Fe}-\mathrm{Al}_{2} \mathrm{O}_{3}$ and $\mathrm{Fe}_{2} \mathrm{O}_{3}-\mathrm{Al}_{2} \mathrm{O}_{3}$ cases. This additional, with respect to the viscosity, damping of the Brownian movement may be of magnetic origin. The magnetic particles moving in the vicinity of metallic particles, here another Fe particles, experienced retardation, similarly to the case of metallic particle moving in magnetic field of another magnetic particles. Certainly, it may be still questionable, if such phenomena observed in macroscopic objects as the consequences of eddy currents, may be applied in nanoscale. If the answer is yes, it leads to the formation of a rather rigid system of Fe magnetic particles, which becomes softer when dissolved by aluminum oxide. The fixed Fe particles indirectly influence the mobility of nonmagnetic $\mathrm{Fe}_{2} \mathrm{O}_{3}$ particles. The proposed model explains the higher mobility of $\mathrm{Fe}_{2} \mathrm{O}_{3}$, and the increase of the mobility of both types of nanoparticles with increasing content of $\mathrm{Al}_{2} \mathrm{O}_{3}$. The further decrease of the mobility for very high amount of $\mathrm{Al}_{2} \mathrm{O}_{3}$ is obvious from the visual inspection of the sediment. The thin layers of the sediments, i.e. these for low $\mathrm{x}$, are very viscous fluids; with increasing their thickness they become nearly solid. Thus, for thick sediments, the dense packing of nanoparticles limits their mobility.

4) The distribution of the velocity is not Gaussian, which points out a distribution of local surroundings of the particles. This may be explained by the 
inhomogeneity in the composition of the sediment. Due to the differences in the sedimentation constant $S$, a quantity used in studying the behavior of colloidal particles; $S=2 r^{2}\left(\rho-\rho^{\prime}\right) / 9 \eta$, where $r$ is the particle radius, $\rho$ and $\rho /$ are densities of particle and medium respectively, and $\eta$ is the medium viscosity, the lower layers of the sediment are enriched in the heaviest component, here Fe.

\section{Conclusions}

The presented results demonstrate the unique ability of Mössbauer spectroscopy in the determination of the mobility of nanoobjects in systems which are very difficult for the study. It should not be forgotten, that the applied technique is only a local complementary research method; the unambiguous conclusions are possible if the studied specimen are well defined. In the studied case, the accurate diagnostics of the investigated system, namely, the sediments composed of magnetic and non- magnetic nanoparticles may confirm the postulated magnetic damping of Brownian movement of iron particles.

\section{References}

[1] J. Szymański, A. Patkowski, A. Wilk, P. Garstecki, R. Holyst, J. Phys. Chem. B 110, 25593 (2006).

[2] J.-S. Lee, J.J. Green, K.T. Love, J. Sunshine, R. Langer, D.G. Anderson, Nano Lett. 9, 2402 (2009).

[3] C. Farokhzad, R. Langer, ACN Nano 3, 16 (2009).

[4] V. Polshettiwar, B Baruwati, R.S. Varma, ACS Nano 3, 728 (2009).

[5] B.J. Berne, R. Pecora, Dynamic Light Scattering, Wiley, New York 1976.

[6] P. Fornal, J. Stanek, A. Wilk, Hyperfine Interact. 185, 81 (2008).

[7] P. Fornal, J. Stanek, Acta Phys. Pol. A 114, 1667 (2008).

[8] J. Stanek, P. Fornal, Hyperfine Interact. 190, 75 (2009). 